\title{
Longitudinal Executive Function and Episodic Memory Profiles in Behavioral-Variant Frontotemporal Dementia and Alzheimer's Disease
}

\author{
Siddharth Ramanan, ${ }^{1}$ Maxime Bertoux, ${ }^{2}$ Emma Flanagan, ${ }^{2}$ Muireann Irish,${ }^{3,45}$ Olivier Piguet, ${ }^{4,5,6}$ John R. Hodges, ${ }^{4,5,6}$ AND \\ Michael Hornberger ${ }^{2}$ \\ ${ }^{1}$ Department of Neurology, Manipal Hospital, Bangalore, India \\ ${ }^{2}$ Norwich Medical School, University of East Anglia, Norwich, United Kingdom \\ ${ }^{3}$ School of Psychology, The University of New South Wales, Sydney, NSW, Australia \\ ${ }^{4}$ Neuroscience Research Australia, Randwick, Sydney, NSW, Australia \\ ${ }^{5}$ ARC Centre of Excellence in Cognition and Its Disorders, Sydney, NSW, Australia \\ ${ }^{6}$ School of Medical Sciences, The University of New South Wales, Sydney, NSW, Australia
}

(Received April 18, 2016; Final Revision July 29, 2016; Accepted September 7, 2016; First Published Online October 18, 2016)

\begin{abstract}
Objectives: With comparable baseline performance on executive functions (EF) and memory between Alzheimer's disease (AD) and behavioral-variant frontotemporal dementia (bvFTD), it is currently unclear if both diseases can be distinguished longitudinally on these measures reliably. Methods: A total of 111 participants (33 AD, 31 bvFTD, and 47 controls) were followed-up annually over a 4-year period and tested on measures of EF, memory, and orientation. Linear mixed-effect models were constructed using disease severity as a nuisance variable to examine profiles of neuropsychological performance decline. Results: At baseline, overlap in terms of cognitive impairment between bvFTD and AD on multiple EF, memory, and orientation measures was present. Longitudinally, only disinhibition (Hayling total errors) appeared sensitive to discriminating AD from bvFTD; however, only after the first annual follow-up. Subgroup analyses on smaller samples revealed comparable profiles on EF tasks at baseline and over time between bvFTD and AD who presented with impaired $\mathrm{EF}$ at presentation, and on memory and orientation tasks between $\mathrm{AD}$ and bvFTD who presented with severe amnesia. Conclusions: Our results replicate previous findings showing only moderate discriminability between AD and bvFTD at clinical presentation on EF and memory measures. More importantly, we also show that longitudinal trajectories strongly overlap for both dementias on these measures. Disinhibition emerged as the sole measure that in the long run was significantly more impaired in bvFTD. Future studies should use tests designed to target cortical regions that are specifically impaired in bvFTD, such as the ventromedial prefrontal cortex, to improve the accurate discrimination of these diseases.
\end{abstract} (JINS, 2017, 23, 34-43)

Keywords: Behavioral-variant frontotemporal dementia, Alzheimer's disease, executive functions, Episodic memory, Orientation, Longitudinal

\section{INTRODUCTION}

Behavioral-variant frontotemporal dementia (bvFTD) is the most common early-onset dementia after Alzheimer's disease (AD) (Piguet, Hornberger, Mioshi, \& Hodges, 2011). In the absence of reliable biomarkers for bvFTD, the distinction between bvFTD and AD relies mostly on clinical characteristics that are considered to be specific to each

Correspondence and reprint requests to: Michael Hornberger, Norwich Medical School, University of East Anglia, Norwich, NR4 7TJ, UK. E-mail: m.hornberger@uea.ac.uk condition. Thus, in their differential diagnosis, revised diagnostic criteria focus on early amnesia as a cardinal cognitive marker of AD (McKhann et al., 2011), while executive dysfunction, in the context of relatively spared episodic memory and visuospatial functions, is emphasized as a core diagnostic criterion for bvFTD in the context of characteristic behavioral changes (Rascovsky et al., 2011). Much depends, therefore, upon caregiver reports, which are not always available or may be unreliable.

Mounting evidence suggests, however, that relying solely on executive dysfunction and memory impairment for clinical differentiation might not be meaningful. Indeed, a "dysexecutive" 
presentation of AD with predominant frontal and temporoparietal atrophy has been extensively described in the last years in biologically/pathologically proven cases. In this atypical presentation of $\mathrm{AD}$, patients present with disproportionately greater executive dysfunction than amnesia (Ossenkoppele et al., 2015; de Souza, Bertoux, et al., 2013). Moreover, studies have also shown that typical AD itself presents with mild to severe executive impairment across a variety of cognitive tests such as verbal fluency, working memory, and attention in which, AD patients may perform comparably to bvFTD patients (Possin et al., 2013; Stopford, Thompson, Richardson, Neary, \& Snowden, 2010; Wong et al., 2016).

Similarly, a growing number of studies have shown that bvFTD can present with a severe episodic memory disturbance similar to AD (Hornberger, Piguet, Graham, Nestor, \& Hodges, 2010; Irish et al., 2011) that cannot be entirely explained by executive dysfunction, but likely also reflects disruption to memory storage and consolidation processes (Bertoux et al., 2014; Irish, Piguet, Hodges, \& Hornberger, 2014). These cognitive findings converge with anatomical findings showing that bvFTD present with atrophy of the hippocampus of analogous severity than in AD (de Souza, Chupin, et al., 2013; Graham et al., 2005; Hornberger et al., 2012) and that severe prefrontal atrophy is observed in AD as well (Ossenkoppele et al., 2015; Wong et al., 2016). This suggests that executive function (EF) and memory impairments may not be specific to bvFTD and AD, respectively, and have limited clinical utility in differentiating uncertain cases of both pathologies.

These results, however, are mostly based on crosssectional studies, and little is understood about how these disease profiles evolve over time. Longitudinal approaches are essential to identify common and disease-specific trajectories of cognitive decline over time (see Kumfor et al., 2014). Currently, it is not clear whether memory and EF longitudinal trajectories of cognitive decline may provide greater clinical utility to accurately distinguish bvFTD and $\mathrm{AD}$ at follow-up. To our knowledge, only one study to date has explored the memory and general cognitive performance of bvFTD longitudinally, revealing limited utility of these cognitive domains in discriminating bvFTD from AD over time (Schubert, Leyton, Hodges, \& Piguet, 2016).

With comparable baseline performance, the latter study showed that longitudinally, bvFTD could be distinguished from $\mathrm{AD}$ only on the basis of two EF (backward digit span and time taken on Trail Making Test-B) and two memory (Doors part A and the memory component of the Addenbrooke's Cognitive Examination - Revised, ACE-R) tests, all measures on which the bvFTD group declined more rapidly than the AD group. One important issue, however, that was not addressed in this study was how varying baseline profiles on EF and memory tasks in bvFTD and AD influence these longitudinal trajectories. Given that some typical AD patients present with severe EF impairment while others do not (Gleichgerrcht, Torralva, Martinez, Roca, \& Manes, 2011; Wong et al., 2016) and that some bvFTD patients can be amnestic while others are not (Bertoux et al., 2014), it is critical to investigate how these different subtypes across both dementias evolve longitudinally.

The current study proposed to fill this gap by examining memory and EF over time in a cohort of AD and bvFTD patients, split further into subtypes based on their EF and memory performance at initial presentation. We also examined longitudinal performance on orientation tasks, which have recently been suggested to discriminate AD from bvFTD at baseline with high reliability (Yew, Alladi, Shailaja, Hodges, \& Hornberger, 2013). Using multi-level linear modeling in a large cohort of AD and bvFTD patients followed up for 3 years, we aimed to identify specific baseline cognitive profiles of $\mathrm{AD}$ and bvFTD patients on these tasks, and explore how disease trajectories of these recently delineated subtypes evolve over time. We also hypothesized that a subgroup of patients in both patient groups would present with severe deficits on EF, memory, and orientation, declining comparably to their comparison groups on longitudinal evaluation.

\section{METHODS}

\section{Participants}

One hundred eleven participants (including $33 \mathrm{AD}$ and 31 bvFTD patients) were recruited from the Frontotemporal Dementia Clinic (FRONTIER) in Sydney. The FRONTIER team is a multidisciplinary clinical research team comprising of neurologists, neuropsychologists, and neuroscientists dedicated to the study of cognitive, behavioral, and brain changes associated with neurodegenerative dementias. All patients met the respective revised diagnostic criteria for $\mathrm{AD}$ and bvFTD (Dubois et al., 2007; McKhann et al., 2011; Rascovsky et al., 2011). We included bvFTD patients with memory impairment if other core diagnostic criteria were present. Patients with no evidence of brain atrophy and disease progression were excluded to avoid potential non-progressive "phenocopy" cases (Hornberger, Shelley, Kipps, Piguet, \& Hodges, 2009).

All AD patients presented with a typical amnesic presentation and signs of AD pathology (including hippocampal atrophy on the MRI) (Dubois et al., 2007), but with an early age of onset. For sake of brevity, throughout this study, we refer to this group as "typical AD." Atypical presentations of AD (e.g., posterior cortical atrophy, corticobasal syndrome, logopenic progressive aphasia) were not included in the study. All patients were assessed by a multidisciplinary team to exclude other neurological/psychiatric etiologies. All caregivers completed the revised Cambridge Behavioral Inventory (CBI-R: Wear et al., 2008) to determine the severity of behavioral symptoms. Disease severity was assessed with the Frontotemporal Dementia Rating Scale (FRS: Mioshi, Hsieh, Savage, Hornberger, \& Hodges, 2010), with lower Rasch scores indicating greater disease severity. Forty-seven healthy controls were selected from the FRONTIER volunteer panel. These participants underwent standard questionnaires for neuropsychiatric symptoms and a structural neuroimaging examination that indicated no atrophy or vascular signs. 
All healthy controls performed within normal range on all cognitive and behavioral measures.

Importantly, all participants were followed-up for a maximum of 3 years with the same neuropsychological assessment performed each year. At baseline, patient groups were matched for sex, age, education, age of disease onset, carer-reported memory impairment, and overall cognitive performance as measured by the ACE-R (all $p$ values >.1).

All participants or their Person Responsible provided written informed consent in accordance with the Declaration of Helsinki. This study was approved by the South Eastern Sydney Local Health District and the University of New South Wales ethics committees. The genetic status of all patients was unknown.

\section{Longitudinal Neuropsychological Assessments}

All participants underwent a comprehensive neuropsychological battery administered annually over 3 years (i.e., baseline +3 annual follow-up periods), including the ACE-R (Mioshi, Dawson, Mitchell, Arnold, \& Hodges, 2006) as a cognitive screening test. They also underwent assessments of (i) EF, including the Hayling test (Burgess \& Shallice, 1997), the Trail Making Test (TMT) parts A and B (Reitan, 1958), verbal letter fluency (with letters F, A, and S: average of three letters used here) as well as forward and backward digit spans; and (ii) orientation and memory processing including temporal and spatial orientation subscales from the ACE-R (sum of temporal and spatial orientation scores reported here), the ACE-R memory subscale (only total score reported here), the Rey Auditory Verbal Learning Test (RAVLT: Schmidt, 1996) (only Long Term Percentage Retention (LTPR) reported here, calculated as delayed recall/final trial learning*100), and the recall of the Rey Complex Figure (RCF: Osterrieth, 1944) after 3-min of delay.

For the sake of comparability to other memory measures, baseline performances on ACE-R total memory $(\max =26)$ and RCF 3-min delayed $(\max =36)$ scores were reported as percentages, by dividing individual scores by maximum possible score and multiplying into 100.

\section{Subgroup Analyses}

\section{Classification of $A D$ patients on executive functioning performance}

As a first step, $\mathrm{AD}$ patients were divided into $\mathrm{AD}$ with spared (AD-SEF) or impaired (AD-IEF) executive functioning. Raw scores at baseline presentation on the four executive functioning tests were transformed to $Z$-scores based on the mean and standard deviation of the control group used in this study. $Z$-scores $\geq 1.5$ (for Hayling A + B error score and TMT B-A time difference) or $\leq-1.5$ (for FAS letter fluency and backward digit span score) were classified to be within the impaired range. In case participants were unable to complete Part B of the TMT or failed to complete it within the prescribed time limit, the maximum Trails-B time score of $300 \mathrm{~s}$ was used to compute their TMT B-A score, in line with previous studies (Wong et al., 2016). In sum, $A D$ patients who were impaired on 0 or 1 of the executive tests were classified as having spared EFs (AD-SEF, $n=20)$ and $A D$ patients who were impaired on $>1$ of the executive tests were classified as having impaired EF (AD-IEF; $\mathrm{n}=13$ ) (Gleichgerrcht, Chade, Torralva, Roca, \& Manes, 2011, Gleichgerrcht, Torralva, et al., 2011; Wong et al., 2016).

\section{Classification of bvFTD patients on memory performance}

All bvFTD patients were classified with "mild" or "severe" memory impairment based on the median score of the group's performance on the ACE-R Total Memory score at baseline presentation. All bvFTD patients with a score of $\geq 19$ on this measure were classified as having "mild" amnesia $(\mathrm{n}=17)$, while all patients with a score $<19$ were classified as having "severe" amnesia $(\mathrm{n}=14)$. For sake of brevity, we hereby refer to these groups as bvFTD-mild and bvFTDsevere, respectively. The same classification was used when evaluating orientation performance. Unlike the AD subgroups that were classified based on four EF tests, the ACE-R Total Memory score (comprising of learning trials, short- and long-delay recalls, recognition, and semantic memory items) in itself comprises of sub-tests from multiple facets of episodic memory, and, therefore, appeared sufficient as a single measure to classify bvFTD patients on.

\section{Statistical Analyses}

Results were analyzed using RStudio v2.13.1. Before any analysis, all variables were plotted and checked for normality of distribution using Shapiro-Wilk tests. Group differences at baseline assessment for demographic and clinical variables were examined using analyses of variance (ANOVAs), and for neuropsychological assessments using analyses of covariance (ANCOVAs) controlling for disease severity as measured by the FRS. Post hoc differences for demographic and clinical variables were computed using the Bonferroni correction, and for neuropsychological data using Tukey's honest significant difference method controlling for disease severity. Effect sizes were indicated using eta-squared values $\left(\eta^{2}\right)$. Correlations coefficients (Pearson's) were computed between baseline EF and memory/orientation performance for $\mathrm{AD}$ and bvFTD groups using $Z$-scores, given scoring differences.

It should be noted that preliminary descriptive analysis for temporal and spatial orientation scores revealed that all controls achieved the maximum possible score (five each) across first, second, and third annual follow-ups, bringing the $\mathrm{SD}$ down to zero at these individual time points. Due to this, $Z$-score computations for patients at individual time points returned scores of infinity. To avoid this infinity value, we allotted all controls an SD of 0.1 at these time points for these measures.

\section{Linear mixed-effect modeling}

Linear mixed-effect models were constructed to examine longitudinal profiles of performance in all groups using 
patient $Z$-scores, relative to control performance at each time point. Only annual follow-ups from baseline assessment were considered. In case of multiple assessments conducted within \pm 6 months of each yearly bracket, the assessment closest to the yearly bracket from baseline was considered. In the model, disease diagnosis was treated as a fixed effect while follow-up time was treated as both a random and fixed effect. A random intercept was included for patient performance and a random slope was added for each follow-up time in the model. Importantly, disease severity as measured by the FRS was added as a covariate. Main effects of follow-up time and an interaction between follow-up time and diagnosis were also modeled. The difference between baseline and first annual follow-up scores were also computed for each measure to form a progression index. Similar models were constructed to analyze $\mathrm{AD}$ and bvFTD subgroup performance.

\section{RESULTS}

\section{Demographics \& Clinical Scores: Baseline Presentation}

\section{Whole group analyses}

Both AD and bvFTD groups were matched for sex, age, education, age of disease onset, carer-reported memory impairments, and general cognitive performance as measured by the ACE-R (all $p$ values $>.1$ ) (Table 1). Disease severity as measured by the FRS and carer-reported behavioral impairments were greater in the bvFTD group than the AD group (both $p<.001$ ). All subsequent analyses were conducted with diseases severity as a nuisance variable to reduce any severity effects in the results.

\section{Subgroup Analyses}

\section{$b v F T D$ subgroups versus $A D$}

bvFTD subgroups were matched with the AD group for sex, age, education, and age of disease onset (Supplementary Table 1). On the ACE-R, the AD group performed intermediately to both bvFTD subgroups $\left(p<.001 ; \eta^{2}=0.20\right)$. Between the subgroups, the bvFTD-severe group had significantly greater number of carer-reported memory complaints $\left(p=.04 ; \eta^{2}=0.15\right)$ and was more impaired than the bvFTD-mild group on the ACE-R $\left(p=.002 ; \eta^{2}=0.26\right)$.

\section{$A D$ subgroups versus bvFTD}

AD subgroups were matched with the bvFTD group for sex, age, education, and age of disease onset. Both AD subgroups had comparable carer-reported memory problems and ACE-R scores as the bvFTD group (all $p$ values $>.1$ ).

Table 1. Demographic, clinical, and neuropsychological data at clinic presentation for AD and bvFTD groups

\begin{tabular}{|c|c|c|c|c|c|}
\hline & $\mathrm{AD}$ & bvFTD & Controls & Group effect & AD vs. bvFTD \\
\hline$N$ & 33 & 31 & 47 & & \\
\hline $\operatorname{Sex}(m: f)$ & $11: 22$ & $7: 24$ & $18: 29$ & $* *$ & n.s. \\
\hline Age (years) & $63.12(16.14)$ & $58.87(16.99)$ & $67.85(5.73)$ & $*$ & n.s. \\
\hline Education (years) & $12.46(3.42)$ & $11.16(2.47)$ & $13.12(2.92)$ & $*$ & n.s. \\
\hline Age of disease onset (years) & $56.45(24.65)$ & $48.16(27.13)$ & - & - & n.s. \\
\hline FRS (Rasch) & $1.07(1.46)$ & $-0.65(1.40)$ & - & - & $* * *$ \\
\hline CBI-R total $(\%)$ & $20.43(12.81)$ & $41.65(16.52)$ & $4.15(4.64)$ & $* * *$ & $* * *$ \\
\hline CBI - R Memory (\%) & $45.10(21.69)$ & $51.74(21.35)$ & $6.95(6.87)$ & $* * *$ & n.s. \\
\hline ACE-R total (100) & $70.66(12.71)$ & $67.76(16.25)$ & 94.79 (2.99) & $* * *$ & n.s. \\
\hline \multicolumn{6}{|l|}{ Executive functions } \\
\hline Backward Digit Span & $5.30(1.55)$ & $4.25(2.37)$ & $8.14(2.56)$ & $* * *$ & n.s. \\
\hline TMT B-A Time Difference (seconds) & $127.12(150.49)$ & $143.65(81.54)$ & $44.41(25.55)$ & $* * *$ & n.s. \\
\hline Hayling A+B Errors & $4.78(3.82)$ & $8.29(5.18)$ & $1.80(2.48)$ & $* * *$ & 0.01 \\
\hline FAS letter fluency & $10.96(4.64)$ & $8.03(4.26)$ & $15.04(4.04)$ & $* * *$ & 0.02 \\
\hline \multicolumn{6}{|l|}{ Orientation and Memory } \\
\hline Orientation - Total (10) & $8.15(1.78)$ & $8.67(1.64)$ & $9.89(0.31)$ & $* * *$ & n.s. \\
\hline ACE-R Total Memory (\%) & $59.32(16.31)$ & $70.59(18.54)$ & $94.27(6.25)$ & $* * *$ & 0.005 \\
\hline RAVLT LTPR $(\%)$ & $40.07(39.18)$ & $55.28(61.65)$ & $85.45(17.97)$ & $* * *$ & n.s. \\
\hline RCF 3-min delayed (\%) & $13.84(13.61)$ & $24.20(18.34)$ & $48.33(17.24)$ & $* * *$ & n.s. \\
\hline
\end{tabular}

Note. Exact p-values reported for post-hoc comparisons on neuropsychological test variables.

$* p<.05$.

$* * p<.01$.

$* * * p<.001$.

n.s., not significant $(p>.1)$.

$\mathrm{AD}=$ Alzheimer's disease ; bvFTD = behavioural variant frontotemporal dementia; CBI-R = Cambridge Behavioural Inventory-Revised; TMT = Trail Making Test; ACE-R = Addenbrooke's Cognitive Examination-Revised; RAVLT LTPR = Rey Auditory Verbal Learning Test Long Term Percentage Retention; RCF = Rey Complex Figure. 
Table 2. Correlation coefficients (Pearson's) for baseline executive and memory/orientation performance for AD and bvFTD groups

\begin{tabular}{|c|c|c|c|c|c|c|c|c|}
\hline & \multicolumn{2}{|c|}{ Orientation } & \multicolumn{2}{|c|}{ Total memory } & \multicolumn{2}{|c|}{ LTPR } & \multicolumn{2}{|c|}{ RCF Recall } \\
\hline & $\mathrm{AD}$ & bvFTD & $\mathrm{AD}$ & bvFTD & $\mathrm{AD}$ & bvFTD & $\mathrm{AD}$ & bvFTD \\
\hline BDS & $0.44 * * *$ & 0.35 & $0.38 *$ & 0.27 & 0.02 & 0.09 & 0.19 & 0.13 \\
\hline TMT B-A & -0.10 & -0.33 & -0.01 & -0.02 & 0.19 & $0.59 * *$ & 0.33 & 0.12 \\
\hline Hayling A + B & -0.16 & -0.20 & -0.31 & -0.39 & -0.22 & 0.16 & -0.20 & -0.29 \\
\hline FAS & 0.04 & 0.36 & 0.27 & 0.33 & 0.00 & -0.03 & 0.20 & 0.19 \\
\hline
\end{tabular}

Note. All correlations computed based on $z$-scores given scoring differences.

$* p<.05$.

$* * p<.01$.

$* * * p<.001$

$\mathrm{AD}=$ Alzheimer's disease; bvFTD = behavioural variant frontotemporal; BDS = Backward Digit Span; TMT B-A = Trail Making Test B-A time difference;

FAS = FAS letter fluency; Total Memory = ACE-R Total Memory score; LTPR = Rey Auditory Verbal Learning Test Long Term Percentage Retention;

$\mathrm{RCF}=$ Rey Complex Figure 3-min delayed recall.

\section{Executive Testing}

\section{Whole group analyses}

At baseline (Table 1), patient groups performed comparably on backward digit span and TMT time difference, while the bvFTD group performed significantly poorer on FAS letter fluency $\left(p=.02 ; \eta^{2}=0.07\right)$ and committed more errors on the Hayling $\mathrm{A}+\mathrm{B}$ tasks than the $\mathrm{AD}$ group $(p=.01$; $\eta^{2}=0.10$ ). Moderately significant correlations between backward digit span performance and orientation and ACE-R total memory performance in the AD group, and large significant correlations between TMT time difference and RAVLT LTPR performance in the bvFTD group were noted (Table 2).

Table 3. Neuropsychological assessment performance: longitudinal evaluation for whole group

\begin{tabular}{|c|c|c|c|c|}
\hline \multirow[b]{2}{*}{ Tests $^{\mathrm{a}}$} & \multicolumn{2}{|c|}{$\begin{array}{l}\text { Main effect of } \\
\text { follow-up time }\end{array}$} & \multicolumn{2}{|c|}{$\begin{array}{c}\text { Interaction between } \\
\text { follow-up time and } \\
\text { diagnosis }\end{array}$} \\
\hline & $\chi^{2 b}$ & $p$ & $\chi^{2 b}$ & $p$-Value \\
\hline Backward Digit Span & 20.89 & $* * *$ & 0.08 & n.s. \\
\hline TMT B-A time difference & 31.95 & $* * *$ & 0.00 & n.s. \\
\hline Hayling A + B Errors & 3.77 & n.s & 4.32 & $*$ \\
\hline FAS letter fluency & 6.82 & $* *$ & 0.01 & n.s. \\
\hline Orientation - Total & 3.36 & n.s & 2.19 & n.s. \\
\hline ACE-R Total memory & 7.71 & $* *$ & 0.66 & n.s \\
\hline RAVLT LTPR & 5.83 & $*$ & 0.00 & n.s \\
\hline RCF 3-min delayed & 2.09 & n.s & 0.27 & n.s \\
\hline ACE-R & 24.14 & $* * *$ & 2.27 & n.s \\
\hline
\end{tabular}

Note.

${ }^{\mathrm{a}}$ All models fitted on Z-scores.

${ }^{\mathrm{b}}$ All degrees of freedom $=1$.

$* p<.05$.

$* * p<.01$

$* * * p<.001$.

n.s., not significant; TMT = Trail Making Test; ACE-R = Addenbrooke's Cognitive Examination-Revised; RAVLT LTPR = Rey Auditory Verbal Learning Test Long Term Percentage Retention; RCF = Rey Complex Figure Test.
Longitudinally (Table 3; Figure 1), the bvFTD and AD groups deteriorated comparably on screening tools and $\mathrm{EF}$ measures except the Hayling $\mathrm{A}+\mathrm{B}$ errors, where bvFTD deteriorated more rapidly than AD. Progression indices, however, suggested that all patient groups displayed a similar pattern of change in performance from baseline to first follow-up on all EF measures. Progression indices and mixed-model findings suggested that after the first follow-up, the bvFTD group declined more rapidly on the Hayling total errors.

\section{$A D$ subgroups versus bvFTD}

At baseline, group effects were noted on all tests (Supplementary Tables 2 and 3). Controls outperformed patient groups on all tests (all $p$ values $<.001$ ). The AD-SEF group performed significantly better than the AD-IEF group on TMT time difference $\left(p=.03 ; \eta^{2}=0.11\right)$ and significantly better than AD-IEF and bvFTD groups on Hayling total errors $(p<.001)$ and FAS letter fluency $\left(p=.002 ; \eta^{2}=0.18\right)$. Importantly, the AD-IEF and bvFTD groups performed comparably on all EF measures (all $p$ values $>.1$ )

Longitudinally, both AD subgroups and the bvFTD group appeared to deteriorate linearly on all EF measures across follow-ups. Progression indices suggested that all patient groups displayed a similar pattern of change in performance from baseline to first follow-up on all EF test measures.

\section{Memory and Orientation}

\section{Whole group analyses}

At presentation (Table 1), controls outperformed both AD and bvFTD groups on all tests (all $p$ values $<.001$ ). Patient groups performed comparably on orientation, RAVLT LTPR and RCF 3-min delay recall (all $p$ values $>.1$ ) while the AD group performed poorer than the bvFTD group on ACE-R total memory $\left(p=.005 ; \eta^{2}=0.12\right)$. 

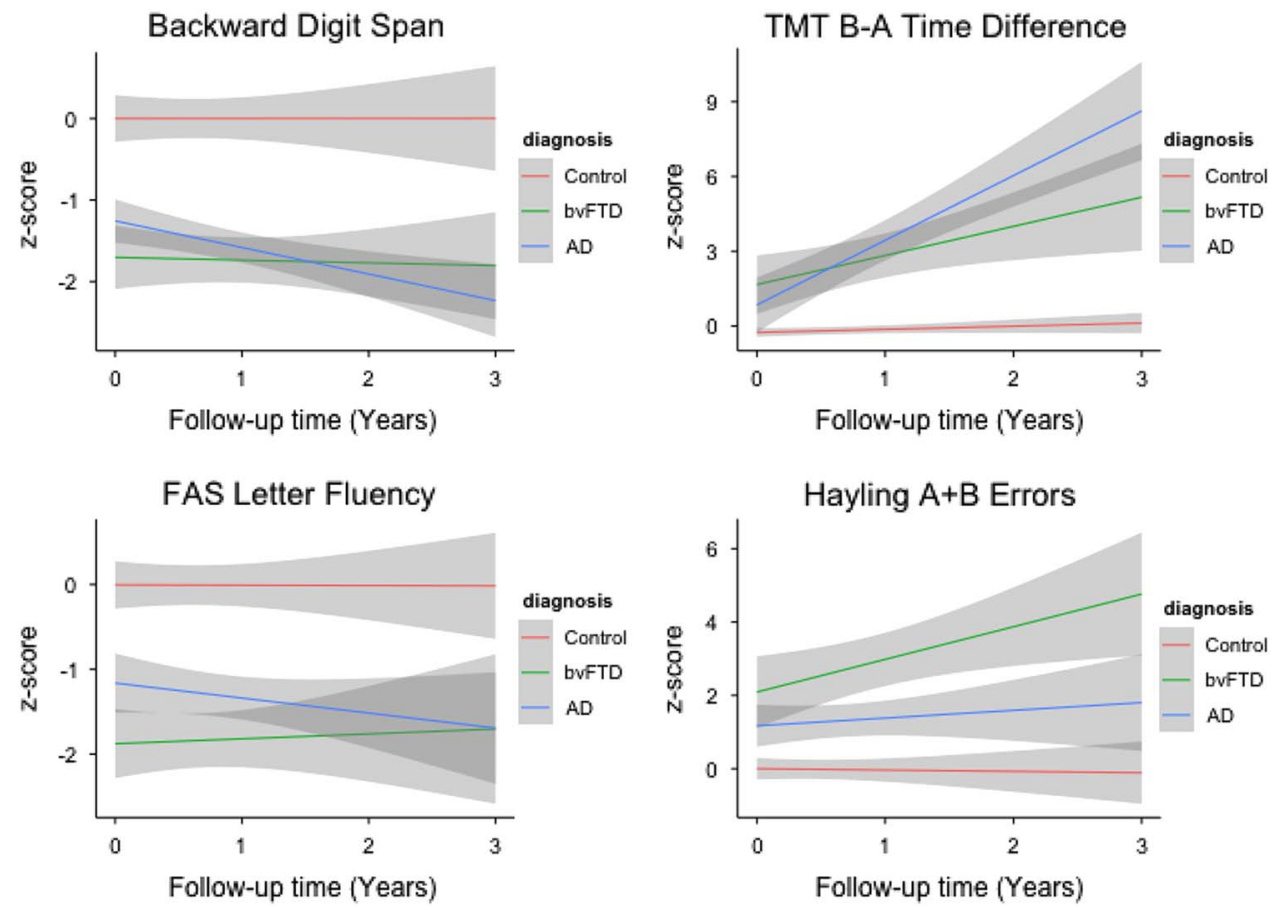

Fig. 1. Longitudinal executive function test performance for whole group.

Note. bvFTD, behavioural-variant frontotemporal dementia; AD, Alzheimer's disease; TMT, Trail Making Test.

Over time, both patient groups deteriorated linearly and comparably across all years of follow-up on all memory and orientation measures (Table 3; Figure 2). Controls showed virtually no change in performance across follow-ups. Progression indices suggested that all patient groups displayed a similar pattern of change in performance from baseline to first follow-up on all memory and orientation test measures.

\section{bvFTD subgroups versus $A D$}

At baseline, group effects were noted on all tests, where controls outperformed all patient groups (Supplementary Tables 2 and 3). The bvFTD-mild group performed better than the bvFTD-severe and AD groups on ACE-R total memory $(p<.001)$, RCF 3-min delayed recall $(p=.01$; $\left.\eta^{2}=0.22\right)$, and total orientation $\left(p=.03 ; \eta^{2}=0.11\right)$. All patient groups performed comparably on the RAVLT LTPR measure. Importantly, the bvFTD-severe and AD groups performed comparably on all memory and orientation measures (all $p$ values $>.1$ ).

Longitudinally, bvFTD subgroups and the AD group appeared to deteriorate linearly on all memory and orientation measures across follow-ups. Progression indices suggested that all patient groups displayed a similar pattern of change from baseline to first follow-up on all memory and orientation measures.

Implicit to our subgroup analyses were the assumptions that the AD subgroups would be impaired on episodic memory and orientation tasks, and that the bvFTD subgroups group would be impaired on EF tasks. These assumptions were confirmed at baseline and on longitudinal evaluation in our analyses and are presented in Supplementary Tables 4 and 5.

\section{DISCUSSION}

The main finding of our study is that decline on standard measures of EF and episodic memory is not specific to bvFTD and $\mathrm{AD}$ at baseline presentation or over time, with the exception of disinhibition. At baseline, bvFTD can present with severe amnesia mimicking AD (Schubert et al., 2016), whereas $\mathrm{AD}$ can present with severe $\mathrm{EF}$ dysfunction mimicking bvFTD (Wong et al., 2016). Longitudinally, the $\mathrm{AD}$ group as a whole declined comparably to bvFTD on EF measures, while the bvFTD group also deteriorated on memory and orientation measures similarly to AD. Furthermore, splitting the patients according to severity in performance at baseline presentation revealed that $\mathrm{AD}$ patients with impaired EF at presentation declined comparably to bvFTD on executive tasks over time, while severely amnesic bvFTD patients appeared to decline on memory and orientation measures comparably to the AD group. In sum, when considering memory/orientation and EF measures, both groups showed little distinction in test performance at initial presentation and over time, and no reliable distinction could be made between these phenotypes and their bvFTD or AD comparison groups.

When facing the differential diagnostic process between $\mathrm{AD}$ and bvFTD, current diagnostic criteria emphasize early impairment of $\mathrm{EF}$ as a cognitive diagnostic marker for bvFTD, while amnesic and disoriented profiles at 
presentation are more indicative of $\mathrm{AD}$ (McKhann et al., 2011; Rascovsky et al., 2011). However, this distinction has been recently challenged as it has been shown that bvFTD patients present with episodic amnesia similar to that observed in AD (Bertoux et al., 2014; Frisch et al., 2013; Hornberger et al., 2010; Irish, Piguet et al., 2014) and that $\mathrm{AD}$ patients at early stages can present with executive dysfunction (Dickerson \& Wolk, 2011; Hutchinson \& Mathias, 2007).

Although a dysexecutive phenotype of $\mathrm{AD}$ has been proposed, where patients present with disproportionately greater executive dysfunction to amnesia (Ossenkoppele et al., 2015), our findings show that typical amnesic AD can also present with comparable EF impairment to bvFTD. We not only show that typical AD present with dichotomous profiles of severity of EF dysfunction at presentation where the AD-IEF group and bvFTD remain comparable (Wong et al., 2016), but also that these profiles remain relatively unchanged over time across most EF tasks. Our findings replicate and extend earlier results to suggest that $\mathrm{EF}$ deficits in $\mathrm{AD}$ appear to go beyond circumscribed impairments in planning and generation tasks (Godefroy et al., 2014), extending to tasks of verbal working memory (Possin et al., 2013) and inhibition as well. Moreover, our findings also highlight that these EF deficits in AD persist and linearly increase across 3 years of follow-up, remaining comparable to bvFTD.

Our memory and orientation results also replicate previous findings showing equivalent episodic memory deficits between bvFTD and AD (Graham et al., 2005; Hornberger et al., 2010; Irish et al., 2014). Previously, memory impairment in bvFTD has been attributed to EF impairments rather than a primary amnesia, supposedly reflecting degeneration of the prefrontal cortex: the classic hallmark of bvFTD (Hornberger \& Piguet, 2012). More recently, however, neuroimaging and pathological studies have shown that bvFTD patients display marked atrophy of key regions involved in memory processing, such as the hippocampus and other structures within the Papez circuit (de Souza, Chupin, et al., 2013; Hornberger et al., 2012; Irish et al., 2014; Rohrer et al., 2015).

Importantly, recent neuropsychological studies have shown that amnesia in bvFTD appears to be a combination of both executive-mediated and storage-based memory impairments (Bertoux et al., 2014; Mansoor et al., 2015; Wong, Flanagan, Savage, Hodges, \& Hornberger, 2014), contradicting the long-held notion that amnesia in bvFTD arises primarily from $\mathrm{EF}$ impairments. In line with this evidence, the current results suggest than bvFTD patients present with similar disorientation and memory impairments as $\mathrm{AD}$. Moreover, this study also reveals that bvFTD show comparable rate of decline to AD over time on those measures. In fact, even mildly amnesic bvFTD patients showed memory impairment persistently declining across 3-years, albeit performing better than severely amnesic bvFTD and AD patients.

With regard to orientation performance, however, our findings at baseline presentation contrast with previous reports (Yew et al., 2013) that suggest that bvFTD can be discriminated from $\mathrm{AD}$ on orientation performance. Additionally, orientation was also one of two measures (the other being RCF delayed recall) to show no effects of deterioration with time, suggesting that patient groups showed relative stability in performance over annual follow-ups on this measure. One possibility for this difference is that we combined performance on temporal and spatial orientation in the current study to give a global orientation score. Indeed, in the study by Yew et al. (2013), AD patients were found to perform significantly worse on temporal than spatial orientation, and combining both measures may have possibly reduced the global score's sensitivity to discriminating these groups.

With regard to memory and EF performance, while Schubert et al. (2016) found two memory and two EF measures to discriminate AD from bvFTD longitudinally, we found only one measure (Hayling total errors) to hold good clinical utility. Few possible reasons for such inconsistencies can be identified. At baseline, our bvFTD and AD patients were less impaired on particular measures (e.g., backward digit span) but more impaired on others (e.g., ACE-R total) as compared to the cohort included by Schubert et al. (2016). Similarly, here we partialled out variance associated with disease severity that could also have possibly rendered to differences between findings between both studies.

Although studies in the past have attempted to better characterize the less-commonly presenting dysexecutive phenotype of $\mathrm{AD}$ in relation to bvFTD and typical $\mathrm{AD}$ (de Souza, Bertoux, et al., 2013; Ossenkoppele et al., 2015), our findings suggest that typical AD patients can present with and progress to show executive dysfunction as severe as that seen in bvFTD (Wong et al., 2016). In fact, further analyses of patients' memory profile revealed that all $\mathrm{AD}$ patients show severe memory impairments as well at presentation, ruling out the possibility of these patients to have a pure dysexecutive atypical presentation of $\mathrm{AD}$ (see Supplementary Tables 4 and 5).

Our study provides initial evidence that except the Hayling total errors measure that emerged relatively sensitive only after the first annual follow-up, EF, memory, and orientation measures fail to distinguish bvFTD from AD across 3 consecutive years of follow-up, questioning the clinical utility of these measures. Instead, we suggest that targeted cognitive tests that differentially stress cortical regions specifically involved in bvFTD (e.g., ventromedial prefrontal cortex) or in $\mathrm{AD}$ (e.g., posterior cingulate cortex) could be particularly useful in discriminating these diseases (Irish et al., 2014).

Several limitations warrant consideration. For example, to better address our research questions, we split and analyzed our patient groups as mutually exclusive while, it is indeed possible to have a subset of patients showing mixed profiles of EF, memory and orientation deficits. Even though attrition over time occurred so not all patients completed all assessments over time (see Supplementary Table 6), despite this, our findings are in line with previous reports in literature. Attrition may, however, have contributed to null findings 

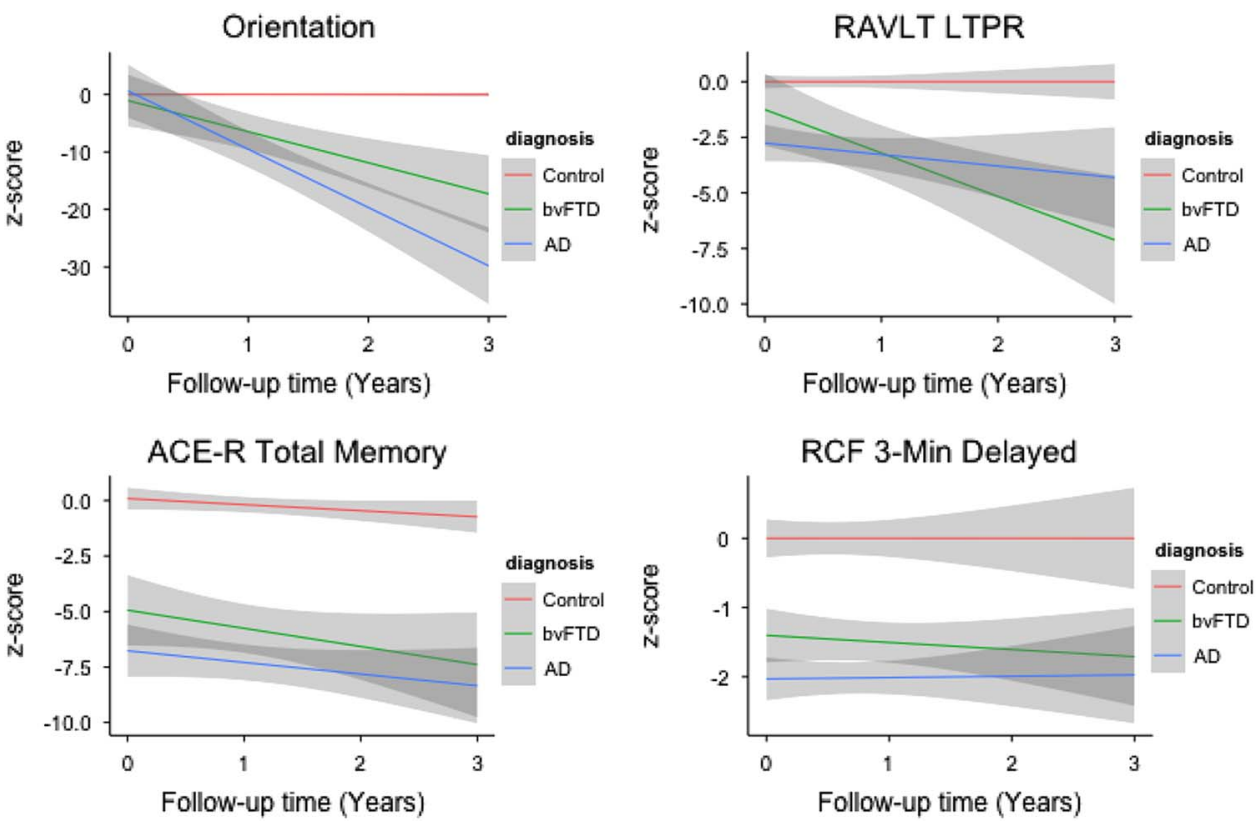

Fig. 2. Longitudinal orientation and memory performance for whole group.

Note. bvFTD, behavioural-variant frontotemporal dementia; AD, Alzheimer's disease; RAVLT LTPR, Rey Auditory Verbal Learning Test Long Term Percentage Retention; ACE-R, Addenbrooke's Cognitive Examination-Revised; RCF, Rey Complex Figure.

as sample sizes reduced considerably with time. Another possibility is that the tasks used in the current study held limited utility in monitoring disease progression in these syndromes.

Another limitation of the current study was that the EF, memory and orientation tests used in the study were part of our diagnostic battery. Our subgroup results also require replication with larger samples. Similarly, addition of the FRS as a nuisance variable in the mixed-model reduced to degrees of freedom to such an extent that it was not possible to provide slopes for all groups. Future studies should address these limitations and focus on developing tests that hold high specificity. It is also imperative for future studies to examine systematic effects of disease severity on test performance, where moderately impaired patients may have more missing data than mildly impaired ones on longitudinal performance. It would also be interesting to investigate the dissociative contributions of frontal and parietal damage early in the presentation of typical $\mathrm{AD}$, as well as the contribution of primary amnesia to deficits on selective EF measures and explore these interactions longitudinally. Furthermore, it would be interesting to correlate cross-sectional and longitudinal performance on memory assessments with underlying pathology in severely amnesic bvFTD patients to investigate if this group would be more likely to pathologically present with greater tau burden in the brain.

Overall, our findings have clear clinical implications. Not only do we show that AD and bvFTD patients can present with similar profiles at baseline on standardized tests of memory, orientation, and EF, but our longitudinal analyses show that both groups deteriorate across these measures at similar rates. Notably, on memory, orientation and EF measures, these groups remain virtually indistinguishable at the first annual follow-up, when diagnostic decisions are commonly revised. Our results, therefore, indicate that episodic memory, EF, and classic spatial and temporal orientation questionnaire assessments that are commonly used in clinical practice hold little clinical utility in the diagnostic distinction of bvFTD and AD at initial presentation and during 3 years of follow-up.

This finding further highlights the need for a revision of the diagnostic criteria of bvFTD, which emphasize the "relative sparing of episodic memory." Our study also highlights that currently used "frontal" tasks are in fact not sensitive to discriminating bvFTD from AD. As bvFTD continues to be diagnosed on presenting behavioral features, there is a crucial need to devise more clinically applicable tasks to assess concomitant changes in social cognition and emotional processing, only few of which (like the mini-SEA: Bertoux, Funkiewiez, O'Callaghan, Dubois, \& Hornberger, 2013) exist that hold enhanced diagnostic sensitivity. Clinicians are better advised to use such social cognition screening tasks for both diseases, which have been shown to be relatively independent of memory performance (Bertoux, de Souza, et al., 2016) and EF (Bertoux, O'Callaghan, Dubois, \& Hornberger, 2016), or novel objective tasks of spatial orientation (Tu et al., 2015) allowing for a reliable distinction of both diseases.

\section{ACKNOWLEDGMENTS}

This work was supported by funding to ForeFront, a collaborative research group dedicated to the study of frontotemporal dementia and motor neuron disease, from the National Health and Medical 
Research Council of Australia (NHMRC) (\#1037746) and the Australian Research Council (ARC) Centre of Excellence in Cognition and its Disorders Memory Node (CE110001021). M.I. is supported by an ARC Discovery Early Career Researcher Award (DE130100463). O.P. is supported by an NHMRC Senior Research Fellowship (APP1103258).

\section{Supplementary material}

To view supplementary material for this article, please visit http://dx.doi.org/10.1017/S1355617716000837

\section{REFERENCES}

Bertoux, M., Funkiewiez, A., O'Callaghan, C., Dubois, B., \& Hornberger, M. (2013). Sensitivity and specificity of ventromedial prefrontal cortex tests in behavioral variant frontotemporal dementia. Alzheimer's \& Dementia, 9, S84-S94.

Bertoux, M., de Souza, L.C., Corlier, F., Lamari, F., Bottlaender, M., Dubois, B., \& Sarazin, M. (2014). Two distinct amnesic profiles in behavioral variant frontotemporal dementia. Biological Psychiatry, 75, 582-588.

Bertoux, M., O'Callaghan, C., Dubois, B., \& Hornberger, M. (2016). In two minds: Executive functioning versus theory of mind in behavioural variant frontotemporal dementia. Journal of Neurology, Neurosurgery, \& Psychiatry, 87, 231-234.

Bertoux, M., de Souza, L.C., O'Callaghan, C., Greve, A., Sarazin, M., Dubois, B., \& Hornberger, M. (2016). Social cognition deficits: The key to discriminate behavioral variant frontotemporal dementia from Alzheimer's disease regardless of amnesia? Journal of Alzheimer's Disease, 49, 1065-1074.

Burgess, P., \& Shallice, T. (1997). The Hayling and Brixton Tests. Test Manual. Bury St. Edmunds, UK: Thames Valley Test Company.

de Souza, L.C., Bertoux, M., Funkiewiez, A., Samri, D., Azuar, C., Habert, M.-O., ... Dubois, B. (2013). Frontal presentation of Alzheimer's disease - A series of patients with biological evidence by CSF biomarkers. Dementia \& Neuropsychologia, 7, 66-74.

de Souza, L.C., Chupin, M., Bertoux, M., Lehéricy, S., Dubois, B., Lamari, F., ... Sarazin, M. (2013). Is hippocampal volume a good marker to differentiate Alzheimer's disease from frontotemporal dementia? Journal of Alzheimer's Disease, 36, 57-66.

Dickerson, B.C., \& Wolk, D.A. (2011). Dysexecutive versus amnesic phenotypes of very mild Alzheimer's disease are associated with distinct clinical, genetic and cortical thinning characteristics. Journal of Neurology, Neurosurgery, \& Psychiatry, $82,45-51$.

Dubois, B., Feldman, H.H., Jacova, C., DeKosky, S.T., BarbergerGateau, P., Cummings, J., ... Scheltens, P. (2007). Research criteria for the diagnosis of Alzheimer's disease: Revising the NINCDS-ADRDA criteria. Lancet Neurology, 6, 734-746.

Frisch, S., Dukart, J., Vogt, B., Horstmann, A., Becker, G., Villringer, A., ... Schroeter, M.L. (2013). Dissociating memory networks in early Alzheimer's disease and frontotemporal lobar degeneration - A combined study of hypometabolism and atrophy. PLoS One, 8, e55251. doi:10.1371/journal.pone.0055251

Gleichgerrcht, E., Chade, A., Torralva, T., Roca, M., \& Manes, F. (2011). Comparing the neuropsychiatric profile of patients with Alzheimer disease who present spared versus impaired executive functioning. Current Gerontology and Geriatrics Research, $2011,514059$.
Gleichgerrcht, E., Torralva, T., Martinez, D., Roca, M., \& Manes, F. (2011). Impact of executive dysfunction on verbal memory performance in patients with Alzheimer's disease. Journal of Alzheimer's Disease, 23, 79-85.

Godefroy, O., Martinaud, O., Verny, M., Mosca, C., Lenoir, H., Bretault, E., \& Roussel, M. (2014). The dysexecutive syndrome of Alzheimer's disease: The GREFEX study. Journal of Alzheimer's Disease, 42, 1203-1208.

Graham, A., Davies, R., Xuereb, J., Halliday, G., Kril, J., Creasey, H., ... Hodges, J. (2005). Pathologically proven frontotemporal dementia presenting with severe amnesia. Brain, 128, 597-605.

Hornberger, M., Shelley, B.P., Kipps, C.M., Piguet, O., \& Hodges, J.R. (2009). Can progressive and non-progressive behavioural variant frontotemporal dementia be distinguished at presentation? Journal of Neurology, Neurosurgery, \& Psychiatry, 80, 591-593.

Hornberger, M., Piguet, O., Graham, A.J., Nestor, P.J., \& Hodges, J.R. (2010). How preserved is episodic memory in behavioral variant frontotemporal dementia? Neurology, 74, 472-479.

Hornberger, M., Wong, S., Tan, R., Irish, M., Piguet, O., Kril, J., ... Halliday, G. (2012). In vivo and post-mortem memory circuit integrity in frontotemporal dementia and Alzheimer's disease. Brain, 135, 3015-3025.

Hornberger, M., \& Piguet, O. (2012). Episodic memory in frontotemporal dementia: A critical review. Brain, 135, 678-692.

Hutchinson, A.D., \& Mathias, J.L. (2007). Neuropsychological deficits in frontotemporal dementia and Alzheimer's disease: A meta-analytic review. Journal of Neurology, Neurosurgery, \& Psychiatry, 78, 917-928.

Irish, M., Hornberger, M., Lah, S., Miller, L., Pengas, G., Nestor, P.J., ... Piguet, O. (2011). Profiles of recent autobiographical memory retrieval in semantic dementia, behaviouralvariant frontotemporal dementia, and Alzheimer's disease. Neuropsychologia, 49, 2694-2702.

Irish, M., Piguet, O., Hodges, J.R., \& Hornberger, M. (2014). Common and unique gray matter correlates of episodic memory dysfunction in frontotemporal dementia and Alzheimer's disease. Human Brain Mapping, 35, 1422-1435.

Kumfor, F., Irish, M., Leyton, C., Miller, L., Lah, S., Devenney, E., ... Piguet, O. (2014). Tracking the progression of social cognition in neurodegenerative disorders. Journal of Neurology, Neurosurgery, \& Psychiatry, 85, 1076-1083.

Mansoor, Y., Jastrzab, L., Dutt, S., Miller, B.L., Seeley, W.W., \& Kramer, J.H. (2015). Memory profiles in pathology or biomarker confirmed Alzheimer's disease and frontotemporal dementia. Alzheimer Disease and Associated Disorders, 29, 135-140.

McKhann, G.M., Knopman, D.S., Chertkow, H., Hyman, B.T., Jack, C.R., Jr., Kawas, C.H., ... Phelps, C.H. (2011). The diagnosis of dementia due to Alzheimer's disease: Recommendations from the National Institute on Aging-Alzheimer's Association workgroups on diagnostic guidelines for Alzheimer's disease. Alzheimer's \& Dementia, 7, 263-269.

Mioshi, E., Dawson, K., Mitchell, J., Arnold, R., \& Hodges, J.R. (2006). The Addenbrooke's Cognitive Examination Revised (ACE-R): A brief cognitive test battery for dementia screening. International Journal of Geriatric Psychiatry, 21, 1078-1085.

Mioshi, E., Hsieh, S., Savage, S., Hornberger, M., \& Hodges, J.R. (2010). Clinical staging and disease progression in frontotemporal dementia. Neurology, 74, 1591-1597.

Ossenkoppele, R., Pijnenburg, Y.A., Perry, D.C., Cohn-Sheehy, B.I., Scheltens, N.M.E., Vogel, J.W., ... Rabinovici, G.D. (2015). The behavioural/dysexecutive variant of Alzheimer's disease: Clinical, neuroimaging and pathological features. Brain, 138, 2732-2749. 
Osterrieth, P. (1944). Le test de copie d'une figure complex: Contribution à l'étude de la perception et de la memoire. Archives of Psychology, 30, 206-356.

Piguet, O., Hornberger, M., Mioshi, E., \& Hodges, J.R. (2011). Behavioural-variant frontotemporal dementia: diagnosis, clinical staging, and management. Lancet Neurology, 10, 162-172.

Possin, K.L., Feigenbaum, D., Rankin, K.P., Smith, G.E., Boxer, A.L., Wood, K., ... Kramer, J.H. (2013). Dissociable executive functions in behavioral variant frontotemporal and Alzheimer dementias. Neurology, 80, 2180-2185.

Rascovsky, K., Hodges, J.R., Knopman, D., Mendez, M.F., Kramer, J.H., Neuhaus, J., ... Miller, B.L. (2011). Sensitivity of revised diagnostic criteria for the behavioural variant of frontotemporal dementia. Brain, 134, 2456-2477.

Reitan, R.M. (1958). Validity of the Trail Making Test as an indicator of organic brain damage. Perceptual and Motor Skills, 8, 271-276.

Rohrer, J.D., Nicholas, J.M., Cash, D.M., van Swieten, J., Dopper, E., Jiskoot, L., ... Rossor, M.N. (2015). Presymptomatic cognitive and neuroanatomical changes in genetic frontotemporal dementia in the Genetic Frontotemporal dementia Initiative (GENFI) study: A cross-sectional analysis. Lancet Neurology, 14, 253-262.

Schmidt, M. (1996). Rey auditory verbal learning test: A handbook. Los Angeles, CA: Western Psychological Services.

Schubert, S., Leyton, C.E., Hodges, J.R., \& Piguet, O. (2016). Longitudinal memory profiles in behavioral-variant frontotemporal dementia and Alzheimer's disease. Journal of Alzheimer's Disease, 51, 775-782.

Stopford, C.L., Thompson, J.C., Richardson, A.M.T., Neary, D., \& Snowden, J.S. (2010). Working memory in Alzheimer's disease and frontotemporal dementia. Behavioural Neurology, $23,177-179$

Tu, S., Wong, S., Hodges, J.R., Irish, M., Piguet, O., \& Hornberger, M. (2015). Lost in spatial translation - A novel tool to objectively assess spatial disorientation in Alzheimer's disease and frontotemporal dementia. Cortex, 67, 83-94.

Wear, H.J., Wedderburn, C.J., Mioshi, E., Williams-Gray, C.H., Mason, S.L., Barker, R.A., ... Hodges, J.R. (2008). The Cambridge Behavioural Inventory revised. Dementia \& Neuropsychologia, 2, 102-207.

Wong, S., Flanagan, E., Savage, G., Hodges, J.R., \& Hornberger, M. (2014). Contrasting prefrontal cortex contributions to episodic memory dysfunction in behavioural variant frontotemporal dementia and Alzheimer's disease. PLoS One, 9, e87778.

Wong, S., Bertoux, M., Savage, G., Hodges, J.R., Piguet, O., \& Hornberger, M. (2016). Comparison of prefrontal atrophy and episodic memory performance in dysexecutive Alzheimer's disease and behavioral-variant frontotemporal dementia. Journal of Alzheimer's Disease, 51, 889-903.

Yew, B., Alladi, S., Shailaja, M., Hodges, J.R., \& Hornberger, M. (2013). Lost and forgotten? Orientation versus memory in Alzheimer's disease and frontotemporal dementia. Journal of Alzheimer's Disease, 33, 473-481. 